\title{
Green-Synthetized Silver Nanoparticles for Nanoparticle-Enhanced Laser Induced Breakdown Spectroscopy (NELIBS) using a Mobile Instrument
}

\author{
F. Poggialini ${ }^{1 *}$, B. Campanella ${ }^{1}$, S. Giannarelli², E. Grifoni ${ }^{1}$, S. Legnaioli ${ }^{1}$, G. \\ Lorenzetti ${ }^{1}$, S. Pagnotta ${ }^{1}$, A. Safi ${ }^{1}$, V. Palleschi ${ }^{1}$ \\ *francesco.poggialini@gmail.com
}

\author{
${ }^{1}$ Applied and Laser Spectroscopy Laboratory, Institute of Chemistry of \\ Organometallic Compounds, Research Area of CNR, Via Giuseppe Moruzzi, 1, 56124 \\ Pisa (Italy) \\ ${ }^{2}$ Department of Chemistry and Industrial Chemistry, University of Pisa, Via Giuseppe \\ Moruzzi, 13, 56124 Pisa (Italy)
}

\begin{abstract}
When compared to other analytical techniques, LIBS shows relatively low precision and, generally, high Limits of Detection (LODs). Until recently, the attempts in improving the LIBS performances have been based on the use of more stable/powerful lasers, high sensitivity detectors or controlled environmental parameters. This can hinder the competitiveness of LIBS by increasing the instrumental setup cost and the difficulty of operation. Sample treatment has proved to be a viable and simple way to increase the LIBS signal; in particular, the Nanoparticle-Enhanced Laser Induced Breakdown Spectroscopy (NELIBS) methodology uses a deposition of metal nanoparticles on the sample to greatly increase the emission of the LIBS plasma. In this work, we used a simple, fast, "green" and low-cost method to synthetize silver nanoparticles by using coffee extract as reducing agents for a silver nitrate solution. This allowed us to obtain nanoparticles of about $25 \mathrm{~nm}$ in diameter. We then explored the application of such nanoparticles to the NELIBS analysis of metallic samples with a mobile LIBS instrument. By adjusting the laser parameters and optimizing the sample preparation procedure, we obtained a NELIBS signal that is 4 times the LIBS one. This showed the potential of green-synthetized nanoparticle for NELIBS applications and suggests the possibility of an in-situ application of the technique.
\end{abstract}

\section{Keywords}

LIBS; Nanoparticle Enhanced LIBS; Silver Nanoparticles; Green Chemistry 


\section{Introduction}

Laser Induced Breakdown Spectroscopy (LIBS) is an atomic emission spectroscopy technique [13] of growing popularity. The technique is widely used in both industrial applications and research laboratories, and several reviews have been published, showing the versatility of LIBS in multiple fields [4-8].

LIBS has the main advantage of the atomic spectroscopy techniques, namely the fast and simultaneous determination of almost all elements, it is easy to operate and can be used to analyze virtually any sample with little to no preparation required. On the other hand, quantitative analysis with LIBS is not easy to perform, due to the need of matrix-matched standards and the relatively low precision of the technique. The sensitivity is also low, when compared with more complex atomic spectroscopy techniques such as ICP-OES. Sensitivity in LIBS is affected by the laser pulse energy, the focalization setup, the type of spectrometer and the collection parameters.

The preferred method for improving LIBS performances has been the implementation of more effective ablation procedures (e.g. multiple laser pulses, controlled atmosphere, vacuum conditions, etc.) or the use of more powerful lasers and high resolution spectrometers [1-3]. However, this approach increases the costs of the instrumental setup and the optimization of the experimental parameters requires good knowledge of both the instrumentation and the principles of Laser-Induced Plasmas (LIPs) and laser-matter interaction.

In the recent years, several studies have explored the possibility of treating the sample in order to enhance the LIBS signal. A review by De Giacomo et. al. presents the most common methods for in situ treatment and the preparation of LIBS samples of different nature [9]. One of most interesting methods proposed by De Giacomo et al. is the Nanoparticle-Enhanced LIBS (NELIBS) technique [10-13]. This technique is being thoroughly discussed in literature and can be summarized as follows. A drop of a solution containing metallic (silver or gold) nanoparticles (NPs) is deposited on the sample surface and the solvent is dried. By focusing the laser pulse onto this deposition, the NPs act as ignitions points for the LIP and the laser electromagnetic field couples with the one induced on NPs surface plasmons, increasing the number of electrons emitted in the plasma. This generates a hotter and more persistent plasma that, in turn, greatly enhances the radiation emission.

This method seems ideal for in situ applications and for simple and fast improvement of LIBS capabilities. It could also be applied in those cases where the instrumental setup cannot be modified, or is not convenient to do it, for example in the case of a commercial LIBS setup. These instruments usually have a set of parameters that are fixed by the manufacturer due to the intrinsic limits that guarantee the portability. Also, these products, are usually intended for customers (e.g. cultural heritage, geology, industry, etc.) that do not necessarily possess the knowledge to optimize the instruments' parameters.

Suitable NP solutions are, however, usually expensive and sometimes not readily available. Thanks to the relative simplicity of these methods and the growing interest for the "green chemistry", the literature is rich in methods for the "green synthesis" of silver NPs, using plant extracts as reducing and capping agents for the reaction with silver nitrate solutions.

In this work, we identified and adapted a procedure for the synthesis of a solution of silver NPs suitable for NELIBS that is "green", low cost, fast and that can be realized with basic laboratory equipment. We tested the performances of the synthesized NPs for NELIBS analysis with a mobile LIBS system developed in our laboratory, and now produced as a commercial instrument for in situ, and we compared them with the enhancement observed using commercially available silver NPs. We also investigated the LODs enhancement for $\mathrm{Zn}$ and $\mathrm{Cr}$ by using NIST Standard Reference Materials (SRMs). 


\section{Materials and Methods}

\section{Materials}

Ground coffee (100\% Arabica) was purchased at the local supermarket. Deionized water from an Elga Purelab Option DV 35 water filtration system was used throughout the study. HPLC grade ethanol (Honeywell, Chromasolv, grade $\geq 99.8 \%$ ) was used for the preparation of the coffee extract. Silver nitrate $\left(\mathrm{AgNO}_{3}\right.$ solid, for analysis) was purchased from Merck.

Commercially available silver nanoparticles (Silver dispersion, aqueous buffer, sodium citrate stabilizer from Sigma-Aldrich) with a diameter of $20 \mathrm{~nm}$ were used as benchmark for the NELIBS enhancement.

In this study, we used copper samples as their metallic and highly conductive nature is ideal for the application of the NELIBS technique. Preliminary measurements and the optimization of the experimental procedure were carried out using commercially available copper sheets. Standard reference materials (SRM 394 Unalloyed Copper I, SRM 395 Unalloyed Copper II, SRM 398 Unalloyed Copper V, SRM 399 Unalloyed Copper VI, National Bureau of Standards) were used for the construction of calibration curves and for the estimation of LODs improvements in NELIBS.

The copper sheets were cut into small pieces, thoroughly cleaned and treated with a solution of $\mathrm{HCl} 1 \mathrm{M}$ (Titolchimica S.p.A.) in an ultrasonic bath in order to remove the superficial oxide layer. After drying at $90^{\circ} \mathrm{C}$ for 30 minutes, the samples were prepared for NELIBS analyses. The SRMs were similarly treated and air-dried before the analyses.

All the glassware was thoroughly washed with deionized water and acetone.

\section{Instrumentation}

UV-Vis measurements were performed using a Perkin Elmer Lambda 25 double beam spectrophotometer. Scans were performed from $300 \mathrm{~nm}$ to $600 \mathrm{~nm}$ (scan rate $240 \mathrm{~nm} / \mathrm{min}, 1 \mathrm{~nm}$ slits).

A Zeiss Libra 120 TEM (courtesy of NEST Institute, Pisa, Italy), equipped with an OMEGA incolumn filter and operating at an accelerating voltage of $120 \mathrm{kV}$, was used for investigating the dimensions of the synthetized nanoparticles. The solutions were deposited on carbon-coated copper grids (Mesh 300).

LIBS and NELIBS analyses were performed using the Modì instrument [14]. Modì is equipped with a Nd:YAG laser (LS2134-D, Lotis Lasers) operating at the fundamental wavelength (1064 $\mathrm{nm}$ ) and delivering two laser pulses of up to $110 \mathrm{~mJ}$ per pulse in $15 \mathrm{~ns}$ FWHM. The delay between the pulses can be adjusted from 0 to $100 \mu \mathrm{s}$, with a maximum repetition rate of $10 \mathrm{~Hz}$. The laser beams are focused into an experimental chamber and the plasma emission is collected by an optical fiber placed at a distance of $1 \mathrm{~cm}$ from the sample and at an angle of $45^{\circ}$ with respect to the laser beam. The ablation spot has a fixed diameter of about $300 \mu \mathrm{m}$. The spectra is resolved using a double spectrometer (AvaSpec-2048-2, Avantes) which operates in the range of $180 \mathrm{~nm}$ to 450 $\mathrm{nm}$ with a resolution of $0.1 \mathrm{~nm}$ and from $450 \mathrm{~nm}$ to $900 \mathrm{~nm}$ with a resolution of $0,3 \mathrm{~nm}$. The acquisition delay can be varied from $1.26 \mu$ s up to several seconds. The acquisition gate is fixed at $2 \mathrm{~ms}$. In this work, we chose to use the system in single pulse mode. The delay of acquisition was maintained at $300 \mathrm{~ns}$. The measurements were carried out with a pulse energy up to $105 \mathrm{~mJ}$.

\section{Silver NPs Synthesis and Recovery}

The method for synthetizing the silver nanoparticle solution is a modified version of the one proposed by Dhand et. al. [15].

The coffee extract was prepared by mixing $2.5 \mathrm{~g}$ of ground coffee and $50 \mathrm{~mL}$ of a solution $1: 1 \mathrm{v} / \mathrm{v}$ of ethanol and water. The solution was kept under vigorous stirring at $60^{\circ} \mathrm{C}$ for one hour and 
cooled down to room temperature. The solid residue was removed by two filtration steps with Whatman No.1 filter paper and the extract was stored in the dark at $4^{\circ} \mathrm{C}$.

For the NPs synthesis, $12.5 \mathrm{~mL}$ of coffee extract were diluted to $25 \mathrm{~mL}$ with deionized water and added to $100 \mathrm{~mL}$ of a $0.05 \mathrm{M}$ solution of $\mathrm{AgNO}_{3}$ in an ultrasonic bath at room temperature, and the agitation was maintained for 15 minutes. A color change in the solution is an indication that the silver nitrate has been successfully reduced to elemental silver and NPs have formed. The flask was then removed from the bath and wrapped in aluminum foil in order to prevent light to interact with unreacted silver nitrate. After an incubation period of two hours at room temperature, the solution was centrifuged at $5600 \mathrm{~g}$ and washed two times with deionized water. It was then transferred in screw-cap PET vials and stored in the dark at $4^{\circ} \mathrm{C}$.

The solution was then freeze-dried and $20 \mathrm{mg}$ of the solid residue were dispersed in $10 \mathrm{ml}$ of deionized water with an ultrasonic bath (30 min). The obtained dispersion was then used for NELIBS analyzes.

\section{Results and Discussion}

\section{Silver NPs characterization}

In order to verify the success of the reaction and the presence of silver NPs in the solution, we measured the UV-Vis absorbance of the $\mathrm{AgNO}_{3}$ solution, the coffee extract and the yellow-orange solution obtained at the end of the reaction. Samples were suitably diluted with deionized water in order to avoid signal saturation.

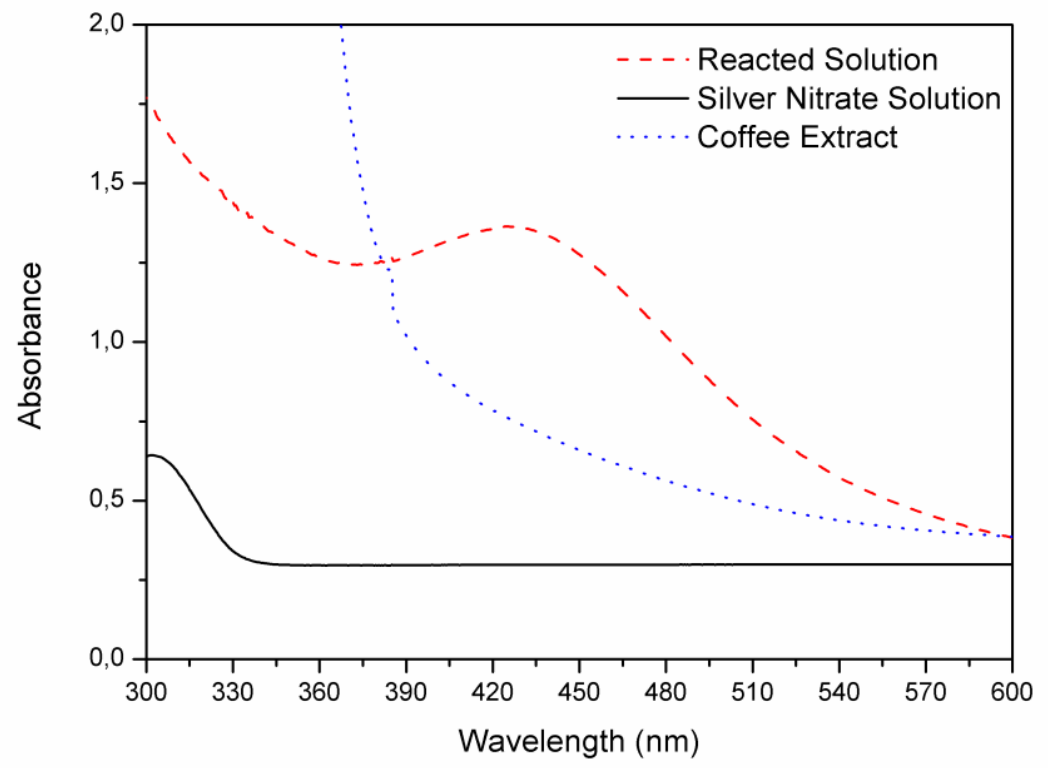

112

Figure 1- UV-Vis spectra of silver nitrate solution (black continuous line), coffee extract (blue dotted line) and the reacted solution (red dashed line)

In Figure 1 can be clearly seen an absorbance band around $435 \mathrm{~nm}$ in the spectrum relative to the reacted solution, that is absent in the other spectra. This is an indication of the presence of silver nanostructures as the band is caused by the surface plasmon resonance (SPR) phenomena, characteristic of nanoparticle solutions. The wavelength value corresponding to the maximum absorbance value in the UV-Vis spectrum could be used to estimate the size of the NPs, even though it is a method prone to errors and interferences [16]. 
TEM images show the presence of spherical and spheroidal nanoparticles, grouped in clusters. We could also observe the presence of seeds and areas where nanostructures are still forming. This suggests that the reaction could be prolonged, even if it could lead to the formation of larger NPs, which are unsuitable for NELIBS analyzes.

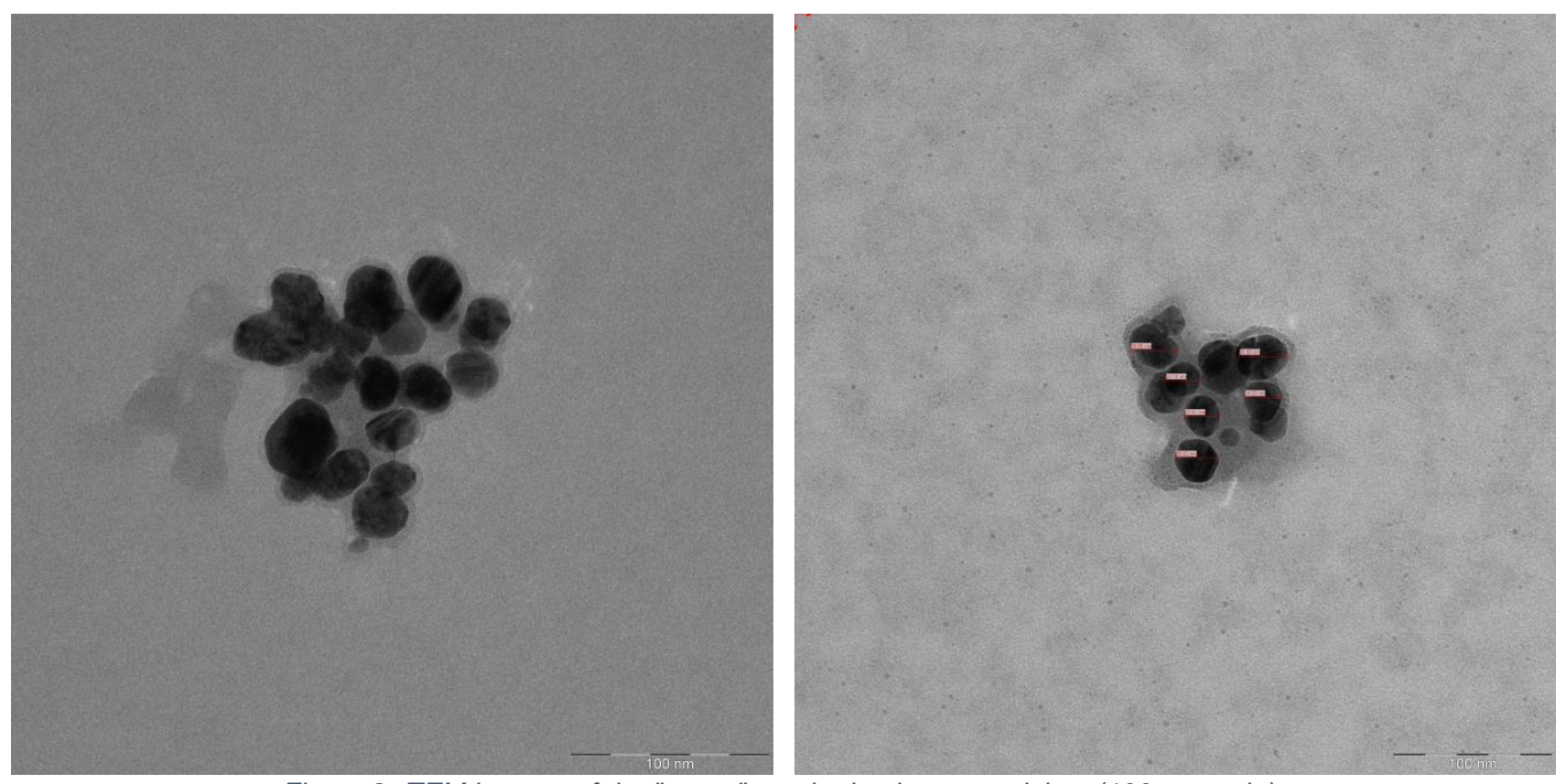

Figure 2- TEM images of the "green"-synthetized nanoparticles. (100nm scale)

From the TEM images we measured an average diameter of the synthetized nanoparticles between 20 and $30 \mathrm{~nm}$. As the solutions appear to be relatively diluted, we decided to prepare a concentrated nanoparticle solution to test the NELIBS performances at various NP concentration. The detailed results are reported in the following sections.

It is important to point out, however, some of the disadvantages related to the "green" synthesis of silver nanoparticles. These methods are mostly used to produce NPs for antimicrobial applications, where precise control of the geometry and the size of the nanostructures is not required and this could be a problem in NELIBS. The NP dimensions can be optimized, but only after a series of tests using various coffee extract or $\mathrm{AgNO}_{3}$ concentrations, reaction times, temperatures, etc. Moreover, with the use of a "green" synthesis, we obtain NPs coated with phytochemicals that can hinder the laser-matter interaction and can negatively affect the enhancement of the LIBS signal. There is also a risk of sample contamination if the NPs are not thoroughly and carefully washed. On the other hand, this kind of approach shows several advantages over the more classical methods of NPs production (i.e. chemical reduction, laser ablation, etc.). It is in line with the current trends of "green chemistry" and it is a relatively inexpensive method that can yield a large quantity of NPs in a short time. It doesn't require aggressive or toxic reagents and its low complexity means that it can be used by personnel with limited laboratory experience. This, coupled with the possibility of using a portable LIBS instrument, makes this method potentially ideal for in situ applications.

\section{LIBS and Sample Treatment Parameters Optimization}

Finding the optimal procedure and instrumental parameters is critical in NELIBS, in order to obtain the highest enhancement possible. In this study, we investigated the effect of the laser pulse energy and the air-drying time on the signal enhancement, expressed as the ratio between NELIBS and LIBS integrated line intensity (EF, Enhancement Factor). For this step, five $5 \mu \mathrm{L}$ droplets of a commercially available $20 \mathrm{~nm}$ silver nanoparticles dispersion were deposited onto a copper sheet for each different measure. Each droplet was analyzed with a single pulse and the 
obtained spectra were averaged. We investigated the non-resonant copper emission lines at 521.8 $\mathrm{nm}(\mathrm{Cu} \mathrm{I})$ and at $578.1 \mathrm{~nm}(\mathrm{Cu} \mathrm{I})$.

The effect of the laser pulse energy on the signal enhancement is illustrated in Figure 3. The lower limit at about $40 \mathrm{~mJ}$ is the lowest energy at which we could obtain reliably a measurable plasma, while the upper limit at about $105 \mathrm{~mJ}$ is the highest energy output of the laser.

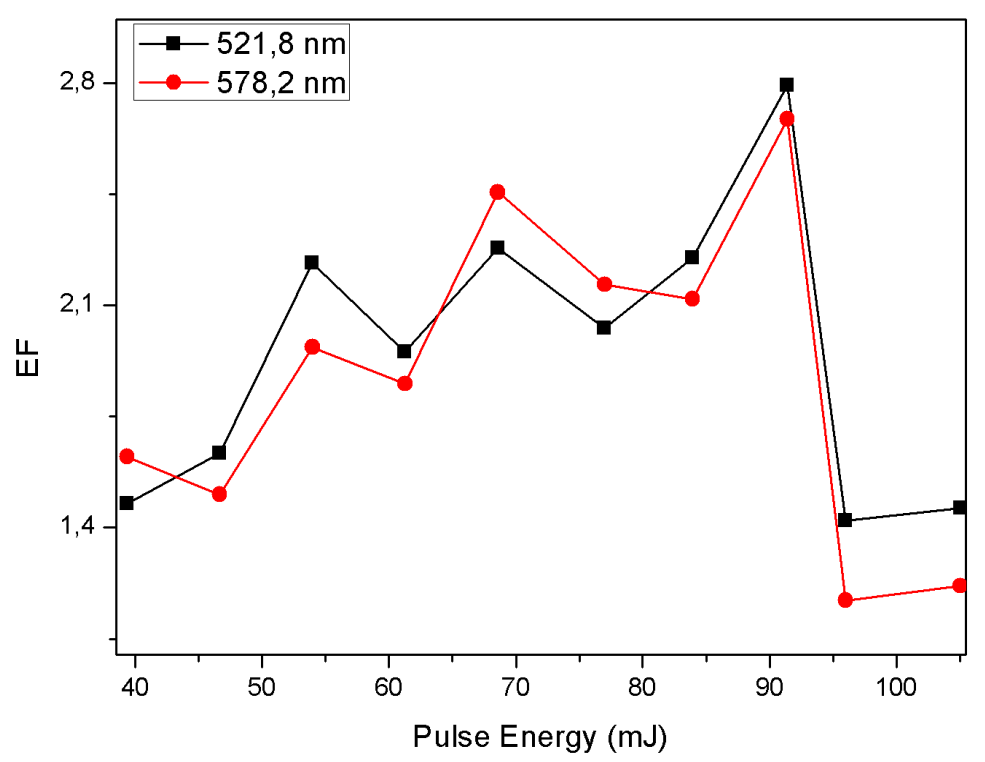

Figure 3 - 20 nm nanoparticles EF as a function of the laser pulse energy

We can see that the EF increases with the pulse energy. The highest enhancement was obtained for a pulse energy of about $90 \mathrm{~mJ}$ and a sharp EF decrease was observed when using higher energies. This is consistent with the work by De Giacomo [12].

Since LIBS performances are greatly affected by the sample surface characteristics, in NELIBS the dryness of the NP deposition is crucial for achieving a good signal enhancement. From a series of preliminary tests, we found that a quick drying of the copper sheets at $90^{\circ} \mathrm{C}$ or with hot air did not yield any appreciable signal enhancement. We then analyzed the depositions after different drying times at ambient temperature (about $25^{\circ} \mathrm{C}$ ) in air. The results are showed in Figure 4. 


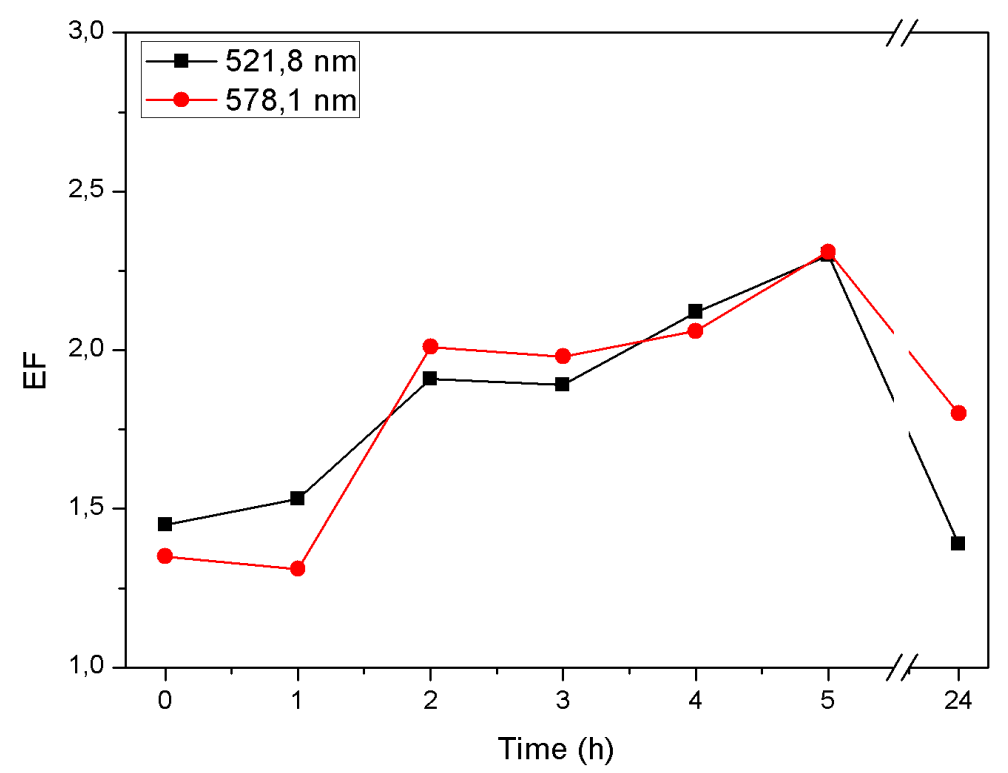

167

Figure 4 - $20 \mathrm{~nm}$ nanoparticles EF as a function of the air-drying time

Similarly to what we observed for the pulse energy, the EF tends to increase with the drying time, up to a maximum at about 5 hours. After that, the EF decreases because of the oxidation of the samples, which is particularly noticeable near the nanoparticle deposition.

Based on these tests, we decided to use a $90 \mathrm{~mJ}$ pulse energy $\left(8.5 \times 10^{13} \mathrm{~W} / \mathrm{m}^{2}\right.$ irradiance) and $4-5$ hours of air-drying for the subsequent NELIBS analyses.

\section{Green Nanoparticles NELIBS Enhancement}

Another critical parameter in NELIBS is the concentration of the NPs on the sample surface and, consequentially, the NPs concentration in the deposited solution. De Giacomo [12] showed the presence of a critical concentration, above which a sharp decrease in NELIBS performances occurs.

In our study, we prepared a dispersion of $2.0 \mathrm{mg} / \mathrm{mL}$ NPs by suspending $20 \mathrm{mg}$ of freeze-dried NPs in $10 \mathrm{~mL}$ of deionized water. From this solution, we prepared 1:5, 1:10, 1:50 and 1:100 v/v diluted dispersions, having a NP concentration of $0.4 \mathrm{mg} / \mathrm{mL}, 0.2 \mathrm{mg} / \mathrm{mL}, 0.04 \mathrm{mg} / \mathrm{mL}$ and 0.02 $\mathrm{mg} / \mathrm{mL}$ respectively. The samples were then prepared and analyzed with the same procedure used for the optimization step and the results are illustrated in Figure 5. 


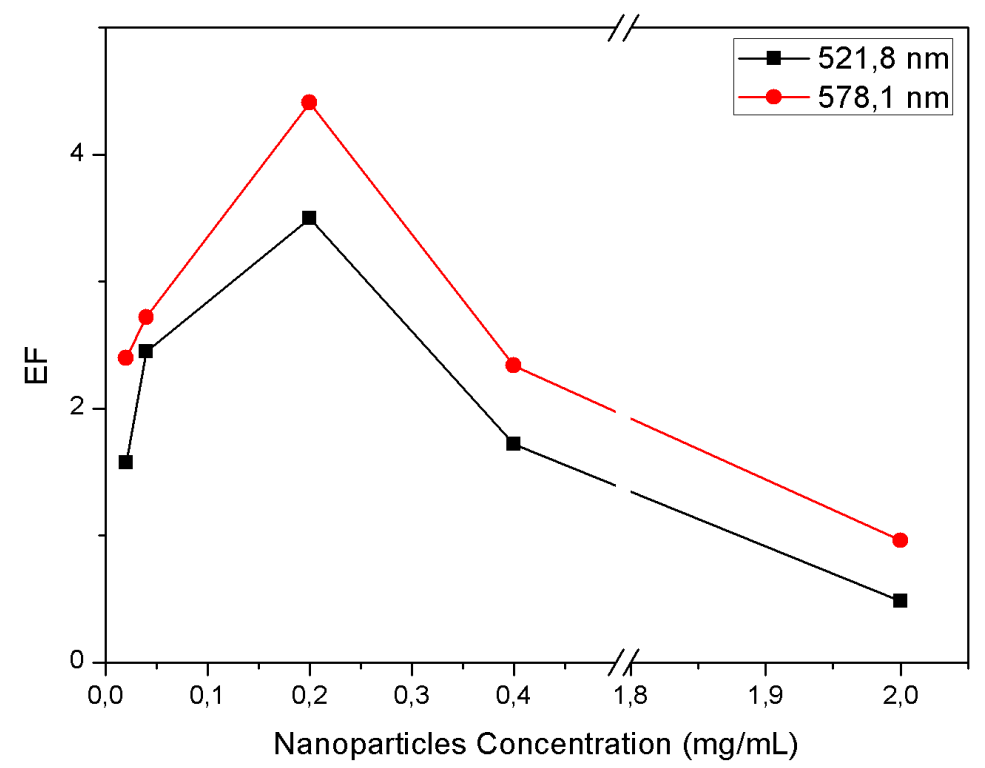

Figure 5 - "Green" nanoparticles EF as a function of the concentration

The highest EF was obtained with the NPs solution at $0.2 \mathrm{mg} / \mathrm{mL}$, resulting in a calculated surface concentration of approximately $1.4 \mathrm{mg} / \mathrm{cm}^{2}$. Using more concentrated solutions resulted in much lower EFs.

It interesting to note how the EF registered when using the synthetized nanoparticles with the optimal procedure and instrumental parameters, is almost two times higher than the one registered when using commercially available silver NPs.

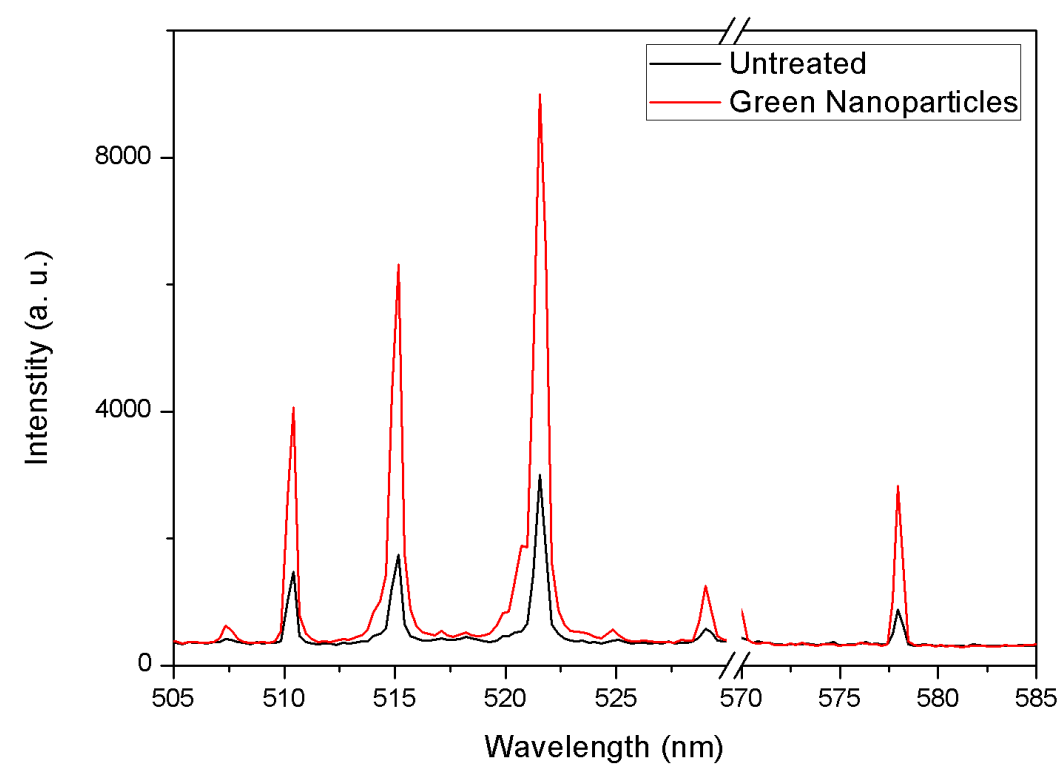

\section{Calibration Curves and LOD Enhancement}


In order to quantify and compare the performances of the synthetized NPs with commercially available ones, we performed a calibration of our system using copper alloy SRMs. Based on preliminary tests, we chose to investigate the emission lines for $\mathrm{Zn} \mathrm{I}(334.5 \mathrm{~nm})$ and $\mathrm{Cr}$ I $(359, .4$ $\mathrm{nm})$. The concentration values for these elements are reported in Table 1.

\begin{tabular}{|c|c|c|}
\hline & $\mathrm{Zn}$ & $\mathrm{Cr}$ \\
\hline SRM 394 & $405 \pm 15$ & $2.0 \pm 0.1$ \\
\hline SRM 395 & $12.2 \pm 0.7$ & $6.0 \pm 0.5$ \\
\hline SRM 398 & $24 \pm 1$ & $0.30 \pm 0.08$ \\
\hline SRM 399 & $45 \pm 3$ & $0.5 \pm 0.1$ \\
\hline
\end{tabular}

Five $5 \mu \mathrm{L}$ droplets of both $20 \mathrm{~nm}$ commercially available NPs and "green" NPs $(0.2 \mathrm{mg} / \mathrm{mL}$ solution) were deposited on the SRM surface and allowed to air dry before the analysis.

NELIBS analyses show that the EF is not the same for copper and the trace elements. This is to be expected as the signal enhancement is related to the upper level energy of the observed transition [10]. Five individual spectra were recorded for each SRM and the integrated line intensities were used to build the curves in Figures 7-8.

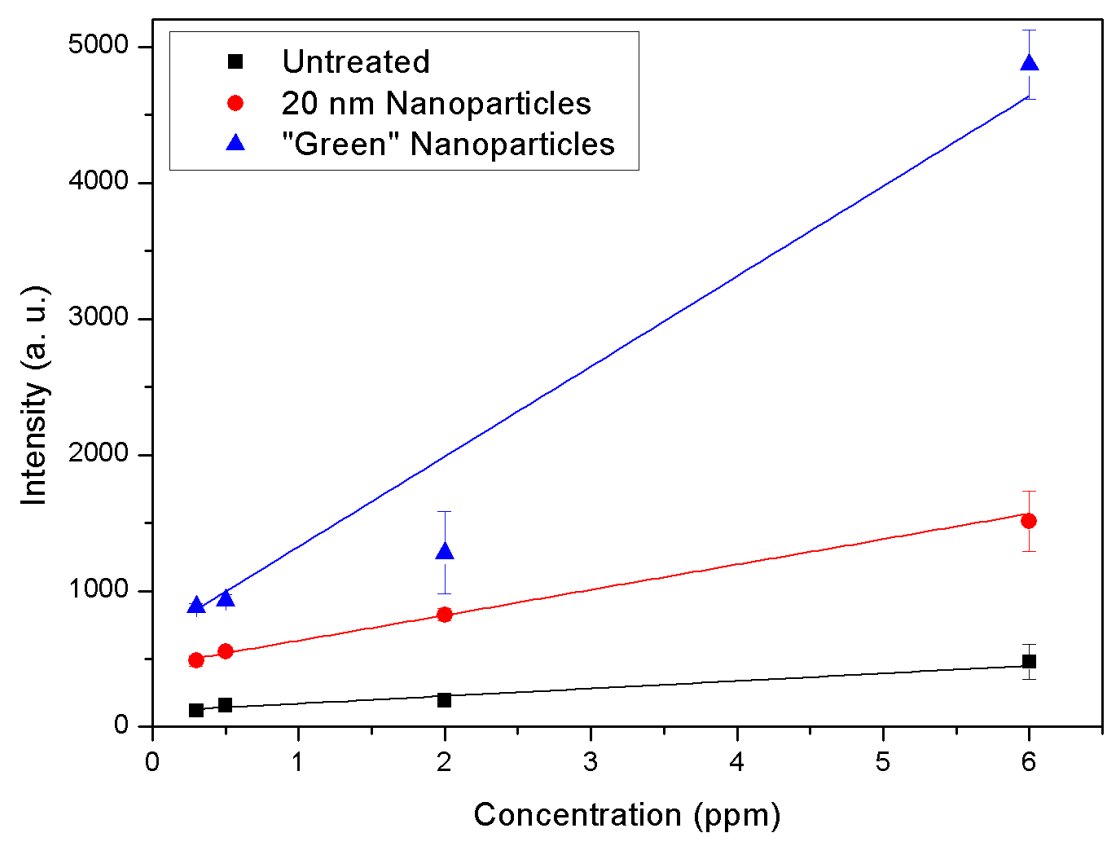




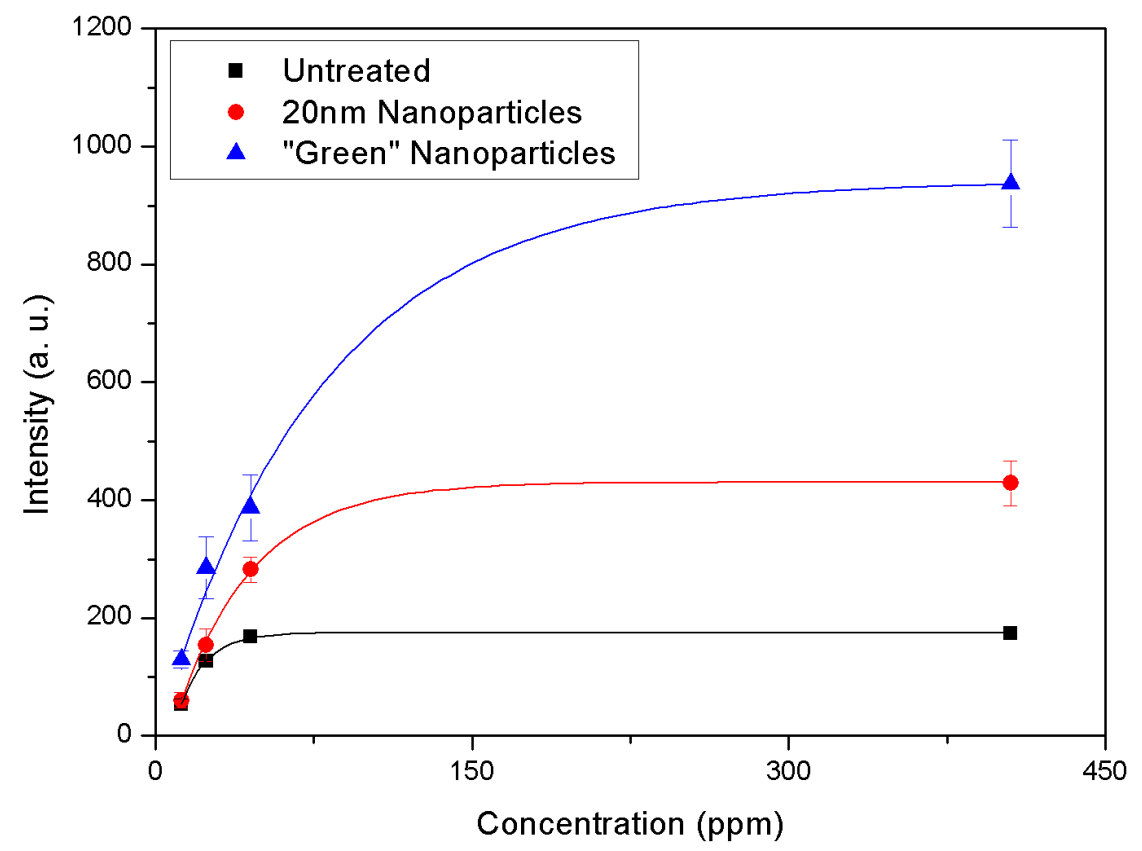

209

Figure 8 - Calibration curves for Zinc

In Table 2 are reported the values of $R^{2}$ for each curve.

\begin{tabular}{cccc}
\hline & & $\mathbf{R}^{2}$ & \\
& Untreated & $\mathbf{2 0} \mathbf{n m}$ NPs & Green NPs \\
\hline $\mathbf{Z n}$ & 0.998 & 0.998 & 0.989 \\
$\mathbf{C r}$ & 0.76 & 0.997 & 0.925 \\
\hline \multicolumn{4}{c}{ Table 2 - Values of $R^{2}$ for the calibration curves }
\end{tabular}

We observed how, in the case of Chromium, that the use of NELIBS increases the quality of the calibration curves. Indeed, this is due to the increased signal intensity, which allow for a better signal to noise ratio and integration of the line intensity. In the case of Zinc, we observed a marked non-linear behavior of the emission intensity for the selected line and we decided to adopt an exponential function for the data fitting, with satisfactory results. It is necessary to point out, however, that the standard deviation values, reported in figures 7-8, are in some cases quite high (i.e. $\approx 25 \%$ ). This is due in part to the surface dependent nature of LIBS and of the used instrumental setup, and in part to the not ideal nature of the SRMs which came in small metal chippings. This greatly hindered the NP deposition and the focalization of the laser onto the samples surface.

From the calibration curves, we estimated the limits of detection (LODs) that are obtainable with NELIBS. By using the relation $L O D \approx 3 \sigma /$ Slope we obtained the values in Table 3.

\begin{tabular}{cccc}
\cline { 2 - 4 } & Untreated & $\mathbf{2 0} \mathbf{~ n m ~ N P s}$ & $\begin{array}{c}\text { Green } \\
\text { NPs }\end{array}$ \\
\hline $\mathbf{C r}$ (ppm) & 1.0 & 0.6 & 0.1 \\
& Table 3-LOD for Chromium &
\end{tabular}

As expected, the LODs are lower in the case of NELIBS analyses. The values also show how, generally, the performances of the "green" NPs are comparable with those of commercially available NP solutions. 
The use of an exponential fitting for Zinc meant that this kind of LOD estimation could not be applied. Even if only 3 points are not enough for a real estimation of the LOD, we tried to use a linear fitting for the low-concentration zone of the calibration curve, in order to obtain a LOD and the results are summarized in Table 4.

\begin{tabular}{cccc} 
& Untreated & $\mathbf{2 0} \mathbf{~ n m ~ N P s}$ & $\begin{array}{c}\text { Green } \\
\text { NPs }\end{array}$ \\
\hline LOD (ppm) & 6.4 & 5.9 & 5.3 \\
$\mathbf{R}^{\mathbf{2}}$ & 0.64 & 0.998 & 0.91 \\
Table 4 - LODs and R2 values for Zinc linear fitting
\end{tabular}

\section{Conclusions}

In this work, we successfully adapted a simple and low-cost method for the "green synthesis" of silver nanoparticles, of comparable morphology with commercially available ones, to the preparation of silver NP solutions suitable for the NELIBS technique.

We were able to optimize the parameters for NELIBS analyses and we established a procedure for the preparation of nanoparticle depositions on the investigated samples.

We registered a signal enhancement using green-synthetized NPs that was higher than the one obtained with commercially available NP solutions. We also investigated and estimated the LODs for NELIBS using the "green" nanoparticles. We found values of $\approx 6 \mathrm{ppm}$ for $\mathrm{Zn}$ and $\approx 0.1 \mathrm{ppm}$ for $\mathrm{Cr}$. We found these LODs to be comparable with those obtained when using commercially available silver NPs.

The results are encouraging and further studies should be carried out in order to improve the quality of the synthetized nanoparticles and obtain a more intense NELIBS signal. It is known from the literature that, in order to obtain the best enhancements in NELIBS (up to 2 orders of magnitude), the use of a laser capable of investigating a much larger area while maintaining a sufficiently high power density is necessary, as the entire NP deposition should be ablated during the laser pulse. The successful application of NELIBS to a mobile, relatively low power LIBS system is, however, an interesting result as it demonstrates the versatility of the NELIBS method, and could be a starting point for further optimization towards in situ NELIBS applications. 
1. S. Musazzi, U. Perini, Laser Induced Breakdown Spectroscopy: Theory and Application, Springer, Heidelberg, 2014, ISBN: 978-3-642-45084-6

2. D. A. Cremers, L. J. Radziemski, Handbook of Laser-Induced Breakdown Spectroscopy, John Wiley and Sons, Ltd., 2006

3. W. Miziolek, V. Palleschi, I. Schechter, Laser-induced breakdown spectroscopy (LIBS): fundamentals and applications, Cambridge: Cambridge University Press, 2006

4. G. S. Senesi, N. Senesi, Laser-induced breakdown spectroscopy (LIBS) to measure quantitatively soil carbon with emphasis on soil organic carbon. A review, Spectrochimica Acta - Part B Atomic Spectroscopy, Vol. 938 (2016) 7-17

5. J. B. Johnson, S.D. Allen, J. Merten, L. Johnson, D. Pinkham, S.W. Reeve, Standoff methods for the detection of threat agents: A review of several promising laser-based techniques, Journal of Spectroscopy, 2014

6. V. Spizzichino, R. Fantoni, Laser Induced Breakdown Spectroscopy in Archeometry: A Review of its Application and Future Perspectives, Spectrochimica Acta - Part B Atomic Spectroscopy, Vol 99 (2014) 201-209

7. A.F.M.Y. Haider, B. Rahman, Z.H. Khan, K.M. Abedin, Survey of the water bodies for ecotoxic metals by laser-induced breakdown spectroscopy, Environmental Engineering Science, Vol. 32(4) (2015) 284-291

8. S. Qiao, Y. Ding, D. Tian, L. Yao, G. Yang, A review of laser-induced breakdown spectroscopy for analysis of geological materials, Applied Spectroscopy Reviews, Vol. 50(1) (2015) 1-26

9. S. C. Jantzi, V. Motto-Ros, F. Trichard, Y. Markushin, N. Melikechi, A. De Giacomo, Sample treatment and preparation for laser-induced breakdown spectroscopy, Spectrochimica Acta - Part B Atomic Spectroscopy, Vol. 115 (2016) 52-63

10. A. De Giacomo, R. Gaudiuso, C. Koral, M. Dell'Aglio, O. De Pascale, Nanoparticle Enhanced Laser Induced Breakdown Spectroscopy: Effect of nanoparticles deposited on sample surface on laser ablation and plasma emission, Spectrochimica Acta - Part B Atomic Spectroscopy, Vol. 98 (2014) 19-27

11. A. De Giacomo, R. Gaudiuso, C. Koral, M. Dell'Aglio, O. De Pascale, NanoparticleEnhanced Laser-Induced Breakdown Spectroscopy of Metallic Samples, Analytical Chemistry, Vol. 85 (2013) 10180-10187

12. De Giacomo, R. Gaudiuso, C. Koral, M. Dell'Aglio, G. Valenza, Perspective on the use of nanoparticles to improve LIBS analytical performance: nanoparticle enhanced laser induced breakdown spectroscopy (NELIBS), Journal of Analytical Atomic Spectrometry, Vol. 31 (2016) 1566-1573

13. De Giacomo, R. Gaudiuso, C. Koral, M. Dell'Aglio, G. Valenza, R. Gaudiuso, Nanoparticle Enhanced Laser-Induced Breakdown Spectroscopy for Microdrop Analysis at subppm Level, Analytical Chemistry, Vol. 88(10) (2016) 5251-5257

14. A. Bertolini, G. Carelli, F. Francesconi, M. Francesconi, L. Marchesini, P. Marsili, F. Sorrentino, G. Cristoforetti, S. Legnaioli, V. Palleschi, L. Pardini, A. Salvetti, Modi: a new mobile instrument for in situ double-pulse LIBS analysis, Analytical and Bioanalytical Chemistry, Vol. 385 (2006) 240-247

15. V. Dhand, L. Soumya, S. Bharadwaj, S. Chakra, D. Bhatt, B. Sreedhar, Green synthesis of silver nanoparticles using Coffea Arabica seed extract and its antibacterial activity, Materials Science and Engineering C, Vol. 58 (2016) 36-43

16. D. Paramelle, A. Sadovoy, S. Gorelik, P. Free, J. Hobley, D. G. Fernig, A rapid method to estimate the concentration of citrate capped silver nanoparticles from UV-visible light spectra, Analyst, Vol 139 (2014) 4855-4861 